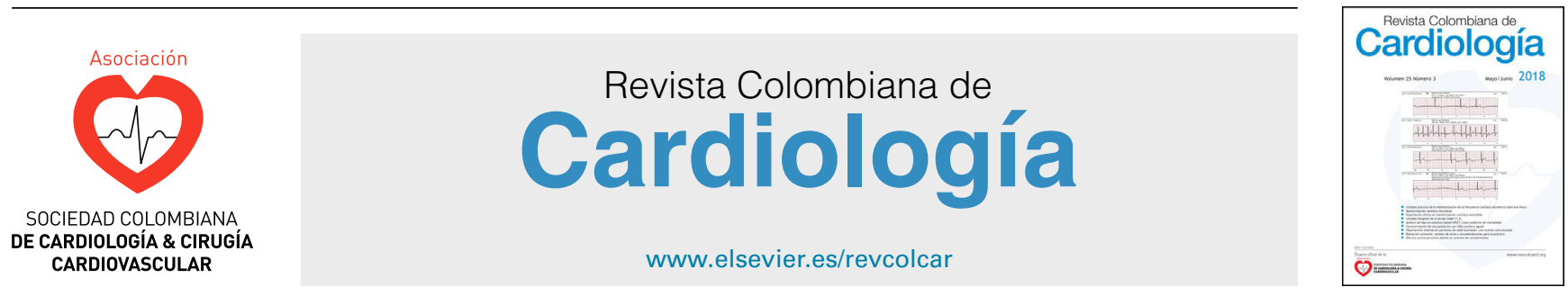

\title{
Bases físicas de la radiación ionizante
}

\author{
Javier Morales-Aramburo* y Jorge Anselmo Puerta
}

Grupo de Física Radiológica, Universidad Nacional de Colombia Sede Medellín, Medellín, Colombia

Recibido el 21 de junio de 2019; aceptado el 24 de enero de 2020

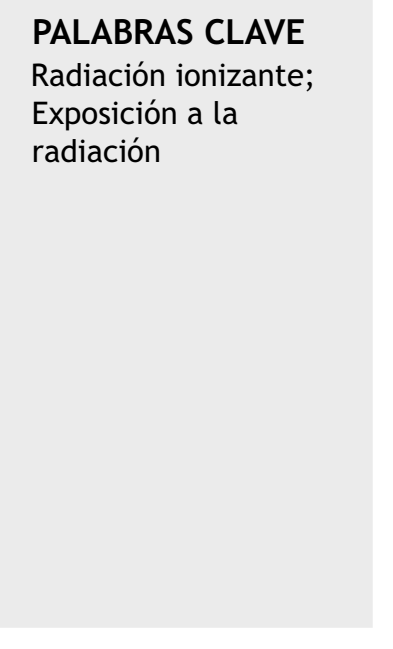

KEYWORDS

lonising radiation;

Exposure to

radiation.

\begin{abstract}
Resumen El electrón, una de las partículas fundamentales de la naturaleza, es el responsable de buena parte de las imágenes médicas actuales, principalmente aquellas que se generan con rayos $X$. Entre las técnicas médicas, basadas en imágenes con rayos $X$, la de mayor desarrollo en la actualidad es la denominada intervencionismo; esta presenta ventajas terapéuticas y de manejo del paciente respecto a anteriores procedimientos como, por ejemplo, la cirugía convencional. Los rayos $X$ son radiaciones ionizantes, y cuando los aplicamos en una actividad positiva para los humanos, como cuando se utilizan en procedimientos guiados por imágenes en cardiología, también incurrimos en un riesgo que debe ser evaluado para controlar que el fiel de la balanza riesgo/beneficio esté siempre inclinado del lado del beneficio. Para describir, medir y evaluar la interacción del campo de radiación $x$ con los tejidos biológicos se definen algunos conceptos, magnitudes y unidades que permiten cuantificar el riesgo de radiación y así poder determinar pautas de comportamiento y protocolos de procedimientos que minimicen los niveles de exposición de pacientes y médicos ocupacionalmente expuestos.

(c) 2020 Sociedad Colombiana de Cardiología y Cirugía Cardiovascular. Publicado por Elsevier España, S.L.U. Este es un artículo Open Access bajo la licencia CC BY-NC-ND (http:// creativecommons.org/licenses/by-nc-nd/4.0/).
\end{abstract}

\section{Physical basis of ionising radiation}

Abstract The electron, one of the fundamental particles of nature, is mainly responsible for the current medical images, particularly those that are produced with X-rays. Among the medical techniques based on images with X-rays, and is currently the most advanced, are found the interventionist procedures. These have therapeutic and patient management advantages as regards previous procedures, such as, conventional surgery. The X-rays are ionising radiations, and when a positive activity is applied by humans, like when image-guided procedures are used in cardiology, there is also a risk that must be evaluated in order to monitor that the needle on the risk/benefit balance is always on the side of the benefits. In order to describe, measure, and evaluate the interaction of the $\mathrm{X}$-ray radiation field with biological tissue, a definition

\footnotetext{
* Autor para correspondencia.

Correo electrónico: jmorales@unal.edu.co (J. Morales-Aramburo).
} 
is needed on the concepts, magnitudes, and units that can help quantify the radiation risk. In this way, behaviour rules and protocols of procedures can be determined that may minimise the radiation exposure of patients and doctors exposed occupationally.

(c) 2020 Sociedad Colombiana de Cardiología y Cirugía Cardiovascular. Published by Elsevier España, S.L.U. This is an open access article under the CC BY-NC-ND license (http:// creativecommons.org/licenses/by-nc-nd/4.0/).

\section{Generación del espectro de rayos $X^{1,2}$}

El campo de radiación $\mathrm{x}$, los rayos $\mathrm{X}$, como los emitidos desde un típico tubo de los utilizados en aplicaciones médicas, consiste de un campo de fotones con una distribución continua de frecuencias $v$ y de energías $E=h v$ donde $h^{*}$ es la constante de Planck. En física de partículas microscópicas, estas presentan la llamada dualidad partícula/onda; así, cada fotón (partícula) de rayos $X$ de energía $E=h v$ tiene asociado un paquete de onda electromagnética de frecuencia media $\bar{v}$ y longitud de onda media $\bar{\lambda} / c$, siendo $c$ la velocidad de la luz (fig. 1). Cuando en el tubo de rayos $X$ los electrones emitidos en el cátodo, y acelerados por un campo eléctrico en su interior, interactúan con los átomos de la pista anódica, hecha de un metal, como tungsteno, molibdeno, plata, cobre, presentan una desaceleración que depende de lo cerca que pasen de los núcleos de los átomos de estos metales; se producen fotones de diferentes frecuencias que en el espectro electromagnético corresponden a los rayos $\mathrm{X}$. En ocasiones, $\mathrm{y}$ dependiendo de la diferencia de potencial, que se establece en el campo eléctrico entre cátodo y ánodo, puede suceder que un electrón que proviene del cátodo al interactuar con el átomo expulse un electrón ligado a la capa más interna, la capa $\mathrm{K}$, produciéndose una vacancia en esta capa que, al ser llenada por un electrón de una capa más externa, la capa $\mathrm{L}$ o la $M$, se emite un fotón $x$ denominado característico del tipo de metal de que está compuesta la pista anódica. Así, por tanto, el espectro de rayos $X$ que sale del tubo y entra al dispositivo llamado colimador, haz útil, tiene la forma típica que se muestra en las figuras 2 y 3; nótese que el espectro comienza en un valor mínimo $\mathrm{E}_{\min }=\mathrm{h} v_{\max }$, donde las energías menores han sido absorbidas en la ventana de berilio (filtración inherente) que poseen los tubos de rayos $X$ en la mayoría de equipos utilizados en la práctica médica.

\section{Formación de imágenes en fluroscopia ${ }^{3,4}$}

Poco después de que Roentgen presentara su descubrimiento de los rayos $X$, la fluoroscopia, o la proyección de imágenes de rayos $X$ en tiempo real, ha estado en uso clínico. Los primeros fluoroscopios consistían simplemente en una fuente de rayos $X$ y una pantalla fluorescente, entre los cuales se colocaba el paciente. Después de pasar por el paciente, el rayo emergente incidía en la pantalla fluorescente produciéndose un brillo visible variable cuyo contraste permitía distinguir órganos y tejidos, que eran observados directamente por el facultativo quien recibía el haz directo exponiéndose a altas dosis de radiación. Los avances en fluoroscopia, en la primera mitad del siglo pasado, consistieron básicamente en encontrar materiales fluorescentes con una mayor eficiencia en cuanto a la conversión de fotones en el rango de frecuencia de los rayos $X$ a fotones del espectro visible. A mediados del siglo pasado se desarrolló el denominado intensificador de imágenes (II) que transformó la práctica médica de manera innovadora, permitiendo una práctica más segura dado que el radiólogo observa la imagen en un monitor de televisión y la radiación que experimenta es la que dispersa el paciente cuando sobre él incide el haz directo. En las décadas más recientes se han introducido los sistemas digitales, en los que los detectores de panel plano (flat panel) son los más populares por sus características, que los hacen más versátiles para el desarrollo de algunas de las prácticas con intervencionismo. A continuación se exponen algunos detalles de los fluoroscopios con II y digital.

\section{Equipos fluoroscópiocos}

Una unidad de fluoroscopia consta básicamente de un tubo de rayos $X$, un generador de voltaje, un intensificador de imagen y un sistema de video. Los tubos de rayos $X$ en fluoroscopia deben tener más alta capacidad de evacuación del calor que un tubo de radiografía convencional, ya que en esta técnica se producen grandes cantidades de calor rápidamente. El generador contiene transformadores de alto voltaje, rectificadores de corriente, un circuito temporizador y selectores de miliamperaje (mA) y kilovoltaje (KVp); y deben ser unidades trifásicas o de alta frecuencia para mejor desempeño en cuanto a la estabilidad de estos parámetros. Para poder utilizar la fluoroscopia en modo cine, es necesario que el generador suministre una alta potencia de salida que posibilite tiempos de exposición cortos, del orden de los milisegundos, y así poder producir una alta tasa temporal de cuadros (frames), lo que es de suma importancia en intervencionismo cardiaco.

El II es un sistema complejo y robusto, encapsulado en un contenedor al vacío. Este convierte el flujo de rayos $X$, modulado por las diferencias de atenuación producidas por los diferentes tejidos y órganos del paciente, que emergen de este e inciden en la denominada ventana de entrada, en una alta fluencia de fotones visible a la salida, en el llamado fósforo de salida. Este proceso se realiza por medio de múltiples capas conversoras de fotones en electrones y viceversa, y una serie de electrodos y lentes electrónicas. A la salida del Il se tiene una imagen óptica que es direccionada por medio de un sistema óptico más cámara de video o CCD, hasta algún dispositivo de despliegue de esta, como un monitor de 


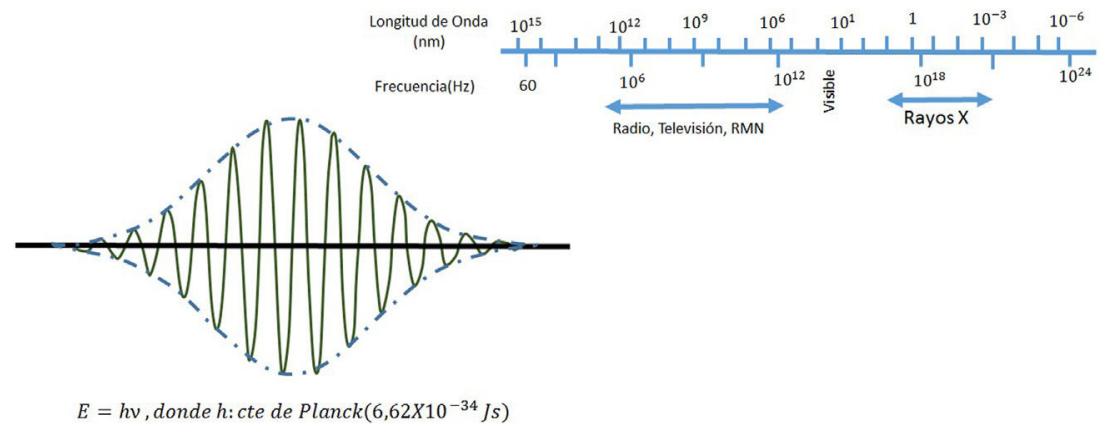

Figura 1 A la izquierda, paquete de onda que representa la localización de un fotón; a la derecha se representa el espectro electromagnético.

televisión o un dispositivo de registro de imágenes. La grabación de imágenes es una parte necesaria de los sistemas fluoroscópicos; varios métodos están disponibles para grabar imágenes desde la salida del II durante la fluoroscopia, que van desde técnicas de registro en película de alta resolución de contraste y baja dosis de radiación, hasta técnicas tipo cine (cinefluorografía), habituales en estudios cardiacos por su alta calidad de imagen, pero que generan altas dosis de radiación (fig. 4).

Las unidades de fluoroscopia modernas incorporan como receptor de imagen el flat panel; este consiste en un arreglo de millones de elementos detectores rectangulares individuales de algunas decenas de micras por lado, son fabricados utilizando tecnología de película delgada sobre sustratos de vidrio de menos de un milímetro de espesor, y se presentan en dos versiones tecnológicas. En la primera, denominada detector digital indirecto, una capa de material centellador, generalmente de yoduro de cesio (Csl), se deposita en la parte superior de un arreglo de transistores de película delgada (TFT), entonces la luz producida en el centellador por los rayos $X$ incide sobre unos fotodiodos que están acoplados a los elementos individuales de la malla de transistores que convierten la luz en carga que es almacenada en capacitores. En la segunda técnica, detector digital directo, utilizan una capa semiconductora gruesa, de silicio o selenio amorfo, sometida a un gran voltaje; cuando los rayos $\mathrm{X}$ son absorbidos en la capa de selenio, pares de carga electrón-hueco se generan y se mueven a los electrodos de polaridad opuesta, y la carga acumulada se almacena en capacitores del arreglo de TFT. En cualquiera de las dos modalidades, los arreglos de TFT de adquisición directa o indirecta, poseen detectores con áreas del orden de los cuarenta centímetros y llegan a tener hasta nueve millones de elementos individuales. En términos generales, en todo sistema digital, la generación de rayos $X$ es realizada por un tubo estándar, y la energía absorbida por el detector se transforma en cargas eléctricas, que se registran, digitalizan y cuantifican en niveles de gris que representan la cantidad de energía de los rayos $X$ depositada en cada punto de digitalización en la imagen resultante.

Los detectores digitales poseen varias ventajas sobre los II, como su mayor tamaño en el área de entrada del detector, menor robutez en su configuración, ausencia de las distorsiones de imagen típicas del II, como son la tipo cojín, barril y $S$, y carencia de problemas, como el velo y el viñeteo asociados con la luz reflejada desde la ventana de salida del II y pérdidas de brillo en la periferia de la imagen, respectivamente. Las tecnologías con receptor de imagen flat panel también permiten nuevas aplicaciones, como la angiografía rotacional y la tomografía con haz cónico (cone beam CT). Sin embargo, los sistemas con II son menos susceptibles al deterioro de la imagen por ruido que el flat panel, particularmente a bajos niveles de exposición (ruido cuántico).

En fluoroscopia se utilizan dos modos de operación denominados exposición continua y exposición pulsada. En la fluoroscopia continua se aplica una corriente continua al tubo y las imágenes se adquieren para una tasa de 30 fotogramas por segundo; el tiempo de adquisición es de 33 milisegundos por imagen. Esto puede conducir a imágenes borrosas de objetos en movimiento como se presenta en cardiología con las palpitaciones del corazón. En el modo pulsado se producen pulsos de radiación cortos e intensos, y es posible controlar su altura (mA), ancho (Dt) y frecuencia $(1 / D t)$. La configuración en modo pulsado ofrece ventajas sobre el modo continuo: dosis más bajas para tasas de pulsos menores a 30 (hasta el $75 \%$ para 7,5/s) y mejora de la calidad de imagen debido a la reducción de la borrosidad por movimiento ya que el tiempo de integración disminuye, así que en modo pulsado se puede "congelar" la imagen de objetos en movimiento y se obtienen imágenes bien definidas de alta calidad. Otra ventaja del modo pulsado es la disminución de la carga del tubo para bajas tasas de pulsos.

La fluoroscopia digital ${ }^{5}$ o computarizada se desarrolló en los años setenta del siglo pasado, y consiste en tomar una imagen fluoroscópica (desde la cámara de televisión o del panel plano) y digitalizar la señal electrónica contenida en esta para enviarla a un computador a fin de realizar el procesamiento en tiempo real. La ventaja de las imágenes digitales es la facilidad y rapidez con la que pueden almacenarse y visualizarse, como también la aplicación de técnicas de substracción digital de imagen, como es común en angiografía, y de congelación de la última imagen, de forma que los intervencionistas las pueden consultar inmediatamente antes de continuar con otro proceso de la exploración o tratamiento.

\section{Magnitudes y unidades de radiación ${ }^{6-8}$}

La radiación es el transporte de energía entre dos lugares de un medio material por partículas tales como los fotones, u otras como por ejemplo partículas beta, o neutrones. Un medio material es un agregado de átomos; en una mol de 
una sustancia hay $6,02 \times 10^{23}$ átomos, de ordinario neutros; es decir, el número de electrones ubicados en las diferentes capas electrónicas, electrones ligados, posee una carga eléctrica negativa que es igual a la carga positiva del núcleo atómico. La denominada radiación ionizante es aquella que tiene la capacidad de sustraer electrones desde los átomos de la materia, inicialmente neutros, con los que interactúan produciendo entonces pares electrón/ion. Los fotones del espectro electromagnético con suficiente energía para ionizar los átomos de la materia son los que poseen alta frecuencia $v$ y corresponden a los rangos denominados $x$ y $\gamma$; estos fotones energéticos al interactuar con la materia eventualmente liberan electrones de los átomos, ionizándolos, por los mecanismos físicos denominados efecto fotoeléctrico y efecto Compton. Entonces cuando los rayos $X$ interactúan con un medio material, como el aire, el agua o tejido biológico, su energía es suficiente para separar electrones de los átomos que componen dichos materiales ionizándolos y, por lo tanto, rompiendo enlaces moleculares. Los tejidos biológicos están compuestos por células, que desde el punto vista químico son agregados de átomos, denominados moléculas, tan especializadas como el ADN. En los tejidos vivos la ionización puede inducir cambios químicos en las células que pueden alterar su material genético (ADN) o causar la muerte celular según el nivel de exposición al que es sometido el tejido biológico. Para bajos niveles de exposición aún es posible que los mecanismos de reparación celular funcionen subsanando adecuadamente el ADN, de lo contrario se producirá una mutación celular que ya no tendrá la información sobre su función orgánica y con el tiempo su reproducción por división celular generará un tumor, posiblemente cancerígeno. En casos de altos niveles exposición se producirá la muerte celular y, por consiguiente, se comprometerá la función del tejido biológico, que, en algunos casos, de acuerdo con la severidad, podrá conducir a la muerte del tejido y comprometerá, por ende, la existencia del individuo para el cual el tejido cumple una función vital. En términos de protección radiológica se habla de efectos estocásticos y efectos tisulares o determinísticos. Los efectos estocásticos se presentan a bajas dosis de radiación, y como su nombre lo indica ocurren al azar; la probabilidad de que se presente un efecto estocástico aumenta con el nivel de exposición a la radiación o dosis de radiación. $\mathrm{El}$ efecto estocástico no tiene, por lo tanto, un umbral para su aparición; un ejemplo típico es la generación de cáncer muchos años después de que células de cierto tejido orgánico mutaran tras ser irradiadas con rayos $X$; también están asociados los efectos hereditarios. En los efectos tisulares o deterministas los daños aparecen dependiendo del nivel de exposición al que se ven sometidos los tejidos biológicos. El efecto será sólo observable clínicamente si la dosis de radiación está por encima de un valor umbral. La magnitud de ese umbral dependerá de la tasa de dosis (es decir, dosis por unidad de tiempo). Con dosis crecientes, por encima del umbral, la probabilidad de ocurrencia se elevará y la severidad del mismo aumentará con la dosis. Estos efectos pueden ocurrir en los procedimientos intervencionistas, en particular, cuando los procedimientos intervencionistas guiados mediante fluoroscopia son complejos y requieren tiempos de radioscopia muy largos o la adquisición de numerosas imágenes. El eritema, "enrojecimiento" de la piel debido a procesos inflamatorios o inmunológicos, puede aparecer pocas horas después de una exposición aguda a la radiación con una dosis en piel de unos 2-3 gray (Gy) para las energías de radiación emitidas por los equipos radiológicos utilizados en las intervenciones. En la práctica real la dosis a la piel en las intervenciones varía según la zona del cuerpo, y es la dosis a la zona más expuesta de la piel (dosis máxima en piel) la que determina la posible aparición de un eritema.

\section{Magnitudes y unidades para dosimetría de radiación ionizante ${ }^{9}$}

En protección radiológica, para estimar el riesgo radiológico en forma cuantitativa, es necesario definir magnitudes y unidades que relacionen los efectos de la radiación en humanos y otras especies a la de la exposición a radiación ionizante.

La exposición $X$ es una cantidad dosimétrica para fotones ionizantes, como los rayos $X$, basada en la capacidad que tienen estos para producir ionización en el aire. El efecto en el tejido u órgano será, en general, proporcional a este efecto en el aire. La exposición es el valor absoluto de la carga total $Q$ de los iones de un signo producido en el aire cuando todos los electrones liberados por fotones por unidad de masa $m$ de aire se detienen completamente en el aire, $X=d Q / d m$.

La unidad de exposición S/* es Coulomb por kilogramo [C kg-1]. La antigua unidad de exposición fue Roentgen [R], $1 \mathrm{R}=2.58 \times 10^{-4} \mathrm{C} \mathrm{kg-1}$. La tasa de exposición es la exposición producida por unidad de tiempo; la tasa de exposición de la unidad SI es la $[(\mathrm{C} / \mathrm{kg}) / \mathrm{s}] \mathrm{o}$, en unidades antiguas [R / s]. En la protección radiológica es común indicar estos valores de frecuencia "por hora" (por ejemplo, R / h).

El Kerma (K). Cuando la radiación de fotones $\mathrm{X}$, con energías como las que se presentan en radiodiagnóstico o intervencionismo, interactúa con un material como un tejido biológico, la energía es transferida a los electrones secundarios que se producen por los diferentes mecanismos físicos, como el efecto fotoeléctrico y la dispersión Compton, así los electrones obtienen cierta energía cinética que irán depositando a lo largo de su trayectoria dentro del material por los mecanismos de ionización y excitación de los electrones atómicos del medio material. Esta energía transferida $\varepsilon$ se llama kerma por su acrónimo en inglés: $\boldsymbol{k}$ inetic $\boldsymbol{e}$ nergy $r$ eleased per unit $\boldsymbol{m}$ ass. En el sistema internacional de unidades la energía se expresa en Julios (J), y por lo tanto el Kerma se expresa como la cantidad de energía $\varepsilon$ liberada por masa de material $m$ irradiado y la unidad se denomina Grey $[\mathrm{Gy}=(\mathrm{J} / \mathrm{kg})]$.

$$
K=\frac{d \varepsilon}{d m}
$$

La dosis absorbida es la magnitud física básica utilizada en protección radiológica, y se define como la energía absorbida $\bar{\varepsilon}$ en un medio, órgano o tejido, dividida por la masa de dicho órgano o tejido,

$$
D=\frac{d \bar{\varepsilon}}{d m}
$$


La unidad asociada a la magnitud de dosis absorbida también es el Grey (Gy). Es la cantidad que debería evaluarse si existe riego de lesiones determinísticas. El medio particular por el que se obtiene D debería especificarse (por ejemplo: aire, piel u otro órgano o tejido específico). Deben especificarse tanto el medio donde se mide la dosis absorbida como las condiciones de irradiación, por ejemplo: dosis absorbida en aire sin retrodispersión (libre en aire) $\left(D_{a, i}\right)$; dosis absorbida en aire con retrodispersión $\left(D_{a, e}\right)$ y dosis absorbida en tejidos (con dispersión de tejido) $\left(D_{t, i}\right)$; en donde $a$ es aire, $i$ es incidente (excluye retrodispersión), e es superficie de entrada (incluye retrodispersión) y $t$ es tejido blando.

A menudo, se utilizan otros términos: "dosis de entrada", "dosis incidente", "dosis de entrada en superficie" y "dosis de entrada al detector"'. Generalmente, se refieren a las dosis absorbidas en aire $u$ otro medio en el punto en que el eje del haz de rayos $X$ ingresa al paciente 0 al detector, pero no siempre está especificado si la cantidad está medida o calculada en aire o algún otro medio, o si la retrodispersión está incluida. Se denomina factor de retrodispersión al cociente entre la dosis en un punto, alcanzado por un haz de Rx, con un determinado material tras él, D, y la dosis en el mismo punto pero eliminando el material, $D_{0}$. Esta información debería estar siempre especificada debido a que la diferencia en el valor numérico de la dosis absorbida puede ser del 30 al 40\%. El factor de retrodispersión en piel para humanos se encuentra en el rango de 1.3 a 1.4 dependiendo, sobre todo, de la edad y la contextura.

Dosis en piel. Es la dosis absorbida media en un área específica de la piel y es una cantidad importante en la evaluación de radiografías o para procedimientos intervencionistas en los que existe riesgo de efectos determinísticos (ICRP, 2001b). La dosis acumulativa máxima en piel (o "dosis máxima en piel, DMP'), es decir, la mayor dosis absorbida, incluida la retrodispersión, es recibida por un área específica de la piel. Esta DPM en la práctica se mide utilizando un arreglo de dosímetros de TLD o película radiocrónica.

La dosis media absorbida en un tejido u órgano $\mathrm{D}_{\mathrm{T}}$ es la energía depositada en el órgano dividida por la masa de ese órgano.

Producto dosis-área. El producto dosis absorbida en aire y el área, DAP, se expresa en $\mathrm{Gycm}^{2}$ y puede estar determinado en cualquier ubicación conveniente en el eje del haz entre la fuente de rayos $X$ y el paciente. El DAP es útil en el control de la dosis para efectos estocásticos a los pacientes y operadores y puede ser medido al utilizar una cámara de ionización de área amplia ubicada en la salida del tubo de rayos $X$. El DAP puede determinarse de manera rutinaria sin interferir con el procedimiento de la imagen si los dispositivos de monitorización adecuados se han instalado en el equipo de rayos $X$. Algunos autores pueden utilizar la cantidad "producto kerma en aire-área, KAP" si la calibración del dispositivo de medición es para Kerma en aire en lugar de dosis absorbida en aire.

La dosis acumulativa $\mathrm{D}_{\mathrm{Ac}}$ (o Kerma en aire acumulativo) es la suma de la dosis (Kerma en aire) en el punto de referencia intervencionista (PRI), durante todos los segmentos de un procedimiento intervencionista. Típicamente se mide en $\mathrm{mGy}$. El punto de referencia intervencionista (PRI) es una ubicación que es representativa de la piel del paciente. Para un equipo de intervención isocéntrico, el PRI está ubicado en el eje central del haz de rayos $X$ a $15 \mathrm{~cm}$ en el lado del tubo de rayos $X$ desde el isocentro. Como el PRI no cambia con la angulación del arco en $\mathrm{C}$, ni con la distancia focoimagen, entonces la $D_{A C}$ es la cantidad que se correlaciona más estrechamente con la dosis en piel en procedimientos guiados por imágenes (figs. 2-10).

Aunque en el pasado las mediciones se han expresado en términos de dosis absorbida en aire, análisis más juiciosos
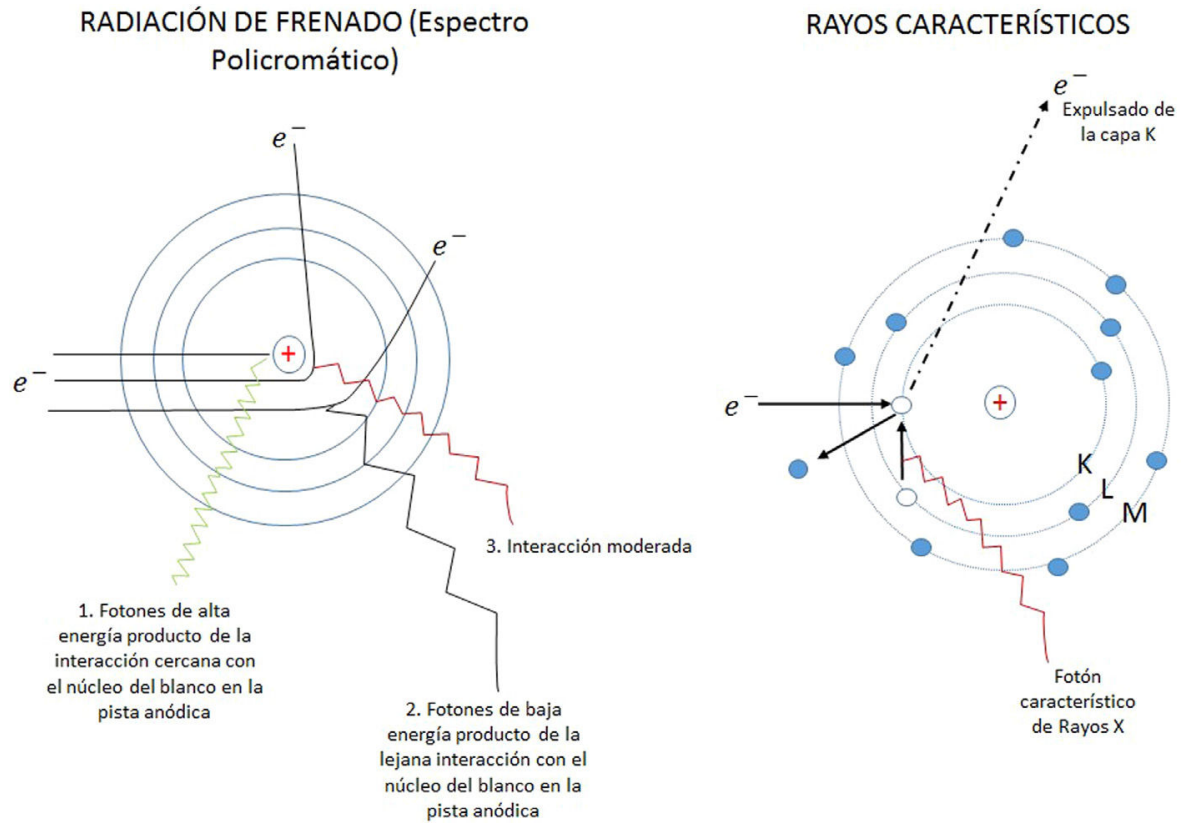

Figura 2 Esquemas que muestran la formación de un espectro de rayos X; a la izquierda se representa el espectro continuo (policromático) de radiación de frenado, y a la derecha la generación de los rayos característicos. 

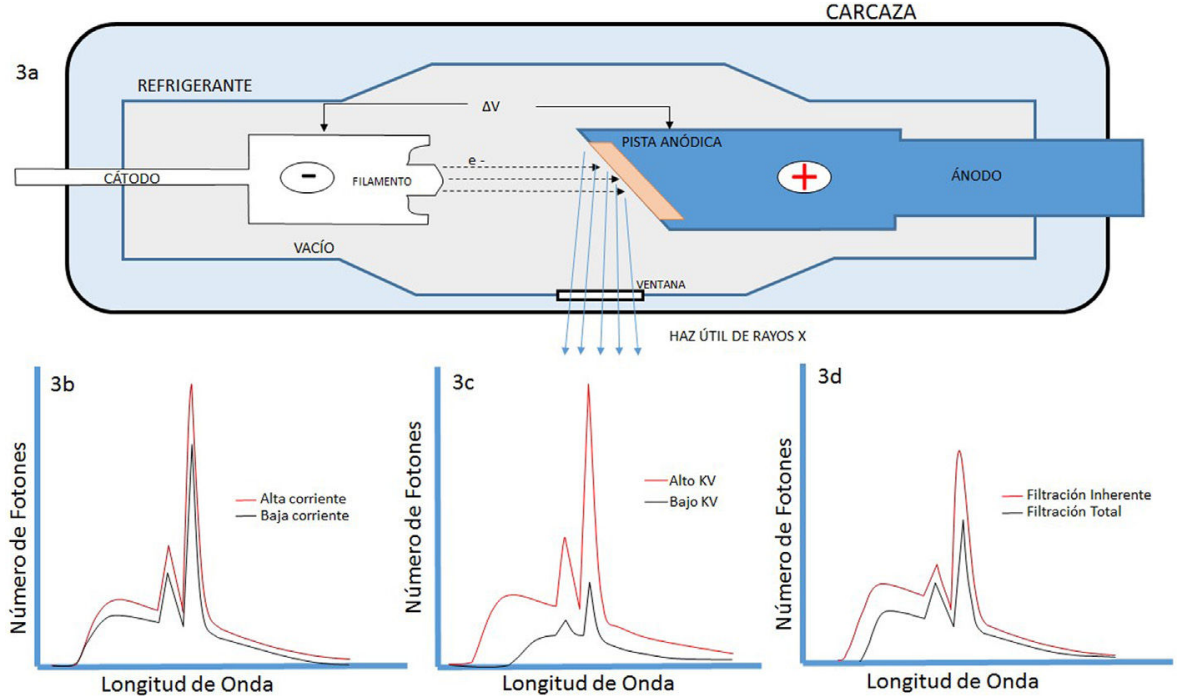

Figura 3 A. Esquema de un tubo de rayos $X$ donde se muestran sus partes y se esquematiza como se producen los rayos $X$ como consecuencia de la interacción de los electrones producidos en el filamento (cátodo), y que son acelerados por el campo eléctrico establecido por la diferencia de potencial $\Delta \mathrm{V}$, con la pista anódica. B, C y D muestran las características principales de los espectros de rayos $X$ que se generan, radiación de frenado y rayos característicos; también se muestran la modificación de estos con el cambio en el miliamperaje mA aplicado en el tubo, el voltaje KV y la filtración.

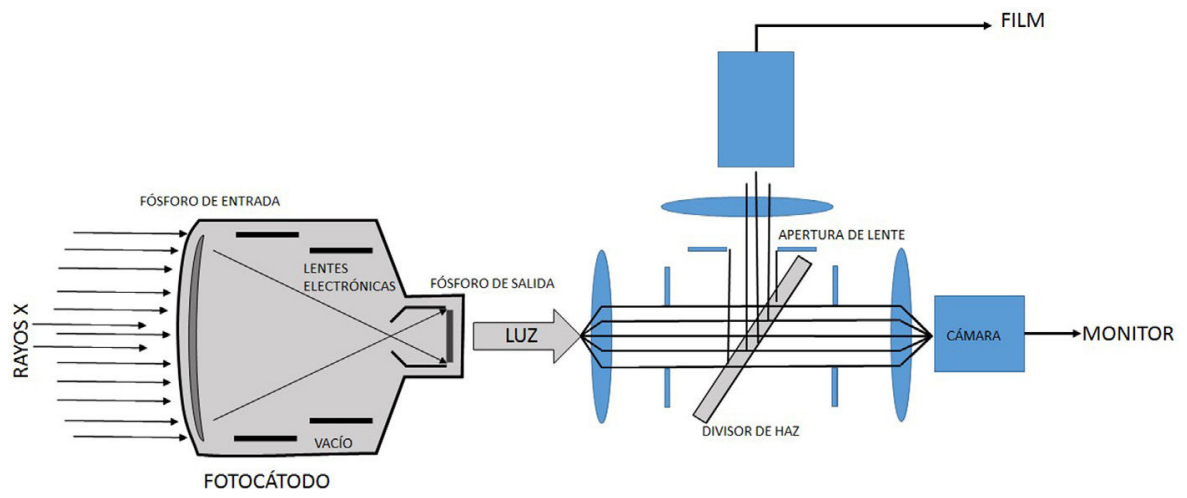

Figura 4 A la derecha: los rayos X que emergen desde el paciente inciden en la ventana de entrada del II, para luego interactuar con el denominado fósforo de entrada, generalmente de cristales de ioduro de cesio, donde se presenta una conversión de fotones $x$ en fotones en el visible; estos a su vez producen electrones al interactuar con el material del fotocátodo por efecto fotoeléctrico. Los electrones así producidos, y distribuidos espacialmente dependiendo de la distribución de rayos X en la ventana de entrada, son enfocados por lentes electrónicas en el fósforo de salida donde se presenta una conversión de electrones en luz. A la izquierda: la luz que emerge del fósforo de salida es recogida por un sistema óptico que la distribuye para el sistema de televisión y sea desplegada en el monitor, o para el sistema de registro, que en los equipos modernos de hemodinámica es un sistema tipo cine.

indican que hay dificultades experimentales para determinar la dosis en aire, particularmente en la cercanía de una interfaz entre dos medios diferentes, y que, en realidad, lo que indican los equipos de dosimetría no es la energía de la radiación absorbida por el aire, sino la energía transferida por la radiación a las partículas cargadas resultantes de la ionización. Por esta razón se recomienda utilizar el kerma en aire en lugar de la dosis absorbida en aire.

Las magnitudes de protección radiológica buscan asegurar que los efectos estocásticos a la salud permanezcan lo más bajo posible y que los efectos tisulares no se produzcan; sin embargo, algunos tipos de radiación son más eficientes que otros para producir efectos estocásticos, y para dar cuenta, de hecho, se ha introducido la magnitud denominada dosis equivalente $H_{T}$, que se define como el producto de la dosis absorbida media en un tejido u órgano multiplicada por un factor de ponderación, $w_{r}$, del tipo de radiación ionizante a que se expone el tejido u órgano. Este factor de ponderación para fotones de radiación $x$ es igual a 1 ; no obstante, para radiación alfa este factor es igual a 20 por su gran capacidad de ionización. La unidad asociada a la dosis equivalente es $\mathrm{J} / \mathrm{kg}$, con el nombre especial "Sievert" (Sv)

$$
H_{T}=W_{R} D_{T}
$$

La probabilidad de aparición de efectos estocásticos depende no solo del tipo de radiación sino también del 


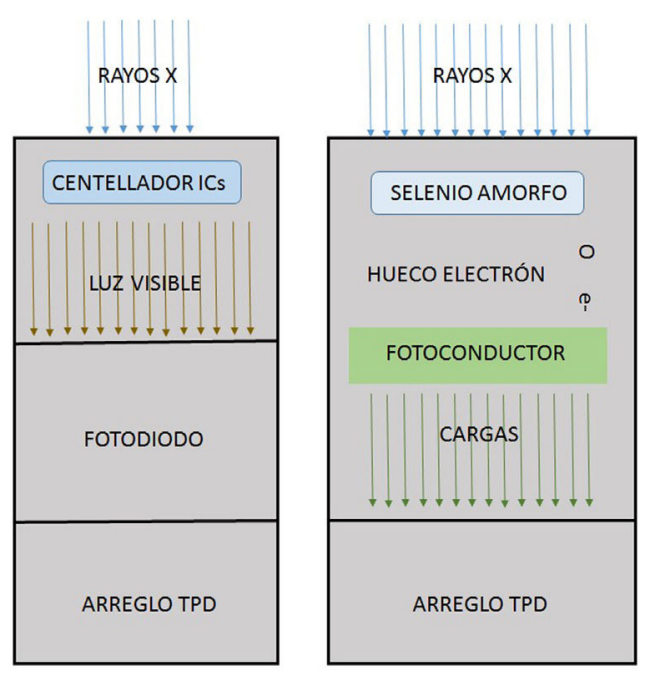

Figura 5 Los detectores digitales se basan en dos tipos de tecnología: la figura de la izquierda muestra el denominado detector digital indirecto en el cual los rayos $\mathrm{X}$ son convertidos en luz visible por medio de un material centellador, generalmente de yoduro de cesio, que es recogido por fotodiodos para conducirlos a los transistores de película delgada (TPD) que se disponen en un arreglo regular de millones de estos. A la derecha se muestra el esquema de trabajo de un detector digital directo; en este un semiconductor amorfo, silicio o selenio, produce un par hueco/electrón cuando sobre él inciden los rayos $\mathrm{X}$; los electrones son recogidos por los colectores de los TPD del arreglo.

órgano considerado. Es decir, no todos los órganos y tejidos del cuerpo humano son igualmente radiosensibles. Por tanto, se consideró apropiado definir una magnitud más, a partir de la dosis equivalente, que tuviese en cuenta la combinación de diferentes dosis en distintos órganos como consecuencia de una irradiación del cuerpo entero. La ICRP introdujo la dosis efectiva con el único objetivo de establecer límites para la protección radiológica ocupacional. La dosis efectiva $(\mathrm{E})$ está dada por la fórmula:

$$
E=\sum T_{W T} H_{T}
$$

Donde $\mathrm{W}_{\mathrm{T}}$ es el factor de ponderación de tejido T. Estos factores de ponderación del tejido, $\mathrm{w}_{\mathrm{T}}$, son más altos para la médula ósea roja, mama, colon, pulmón, estómago, y

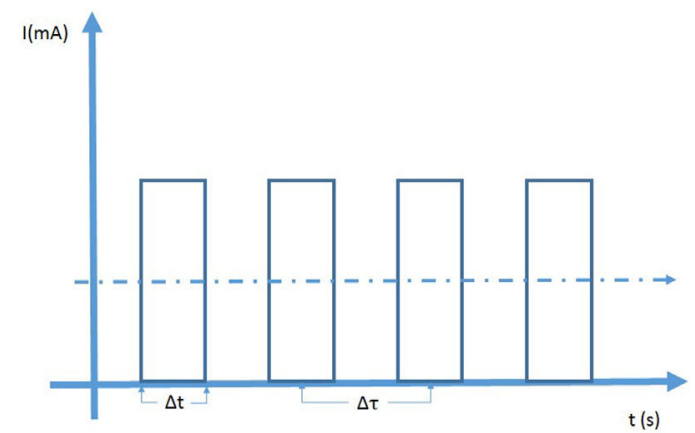

Figura 7 A la izquierda se observa la representación de la fluoroscopia en modo de pulsos. La altura de los pulsos de corriente se puede controlar donde, por lo general, el máximo está alrededor de los $25 \mathrm{~mA}$. El ancho de los pulsos $\Delta \mathrm{t}$ y el tiempo entre pulsos consecutivos $\Delta \tau$ puede ser ajustado. La tasa de dosis depende de la dosis por pulso, su altura su ancho y del número de pulsos por segundo. A la derecha.

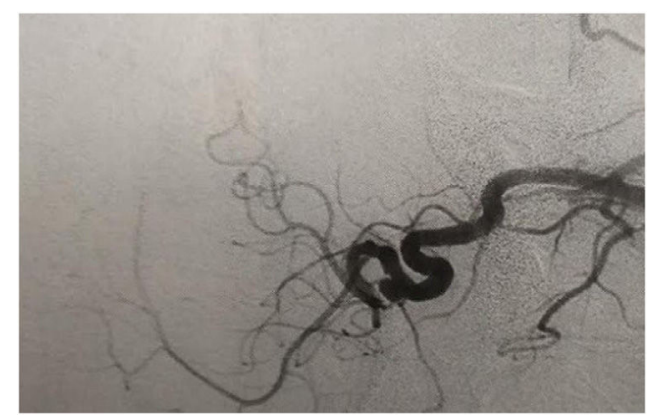

Figura 8 La angiografía por sustracción digital es una técnica en la cual se registra una imagen de la anatomía del paciente y se almacena en la memoria del computador, llamada máscara; después de inyectarle al paciente un agente de contraste radiopaco se van adquiriendo nuevas imágenes que también son almacenadas. La imagen máscara se resta de las imágenes que contienen el agente de contraste y se despliega en el monitor, suministrando una imagen con excelente detalle de la anatomía vascular.

más bajos para hueso cortical, salival, glándulas, cerebro y piel. La dosis efectiva es útil en los estudios de dosimetría al paciente para fines comparativos. No resulta apropiado utilizar dosis efectivas para la estimación del riesgo en la dosimetría al paciente ya que la caracterización de un
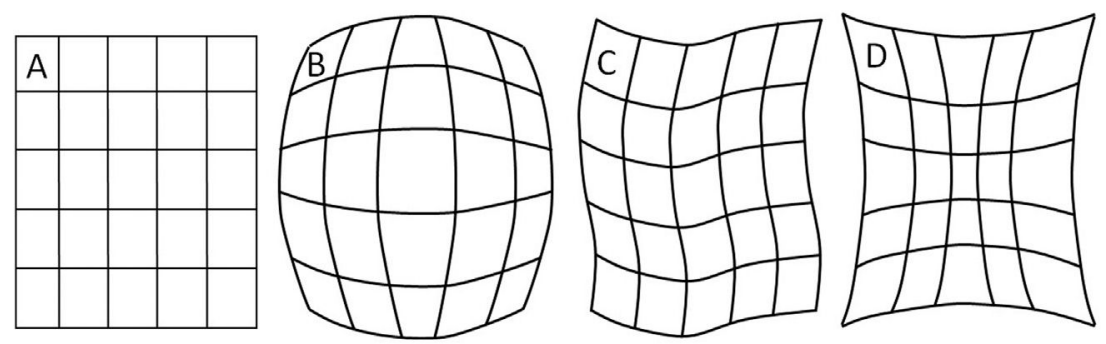

Figura 6 Representación de las distorsiones típicas de los II y que no se presentan con los detectores de panel plano; en A se muestra el objeto de prueba que consiste en una malla rectangular, en B, C y D se muestran las distorsiones denominadas tipo "barril", tipo S y tipo cojín. 


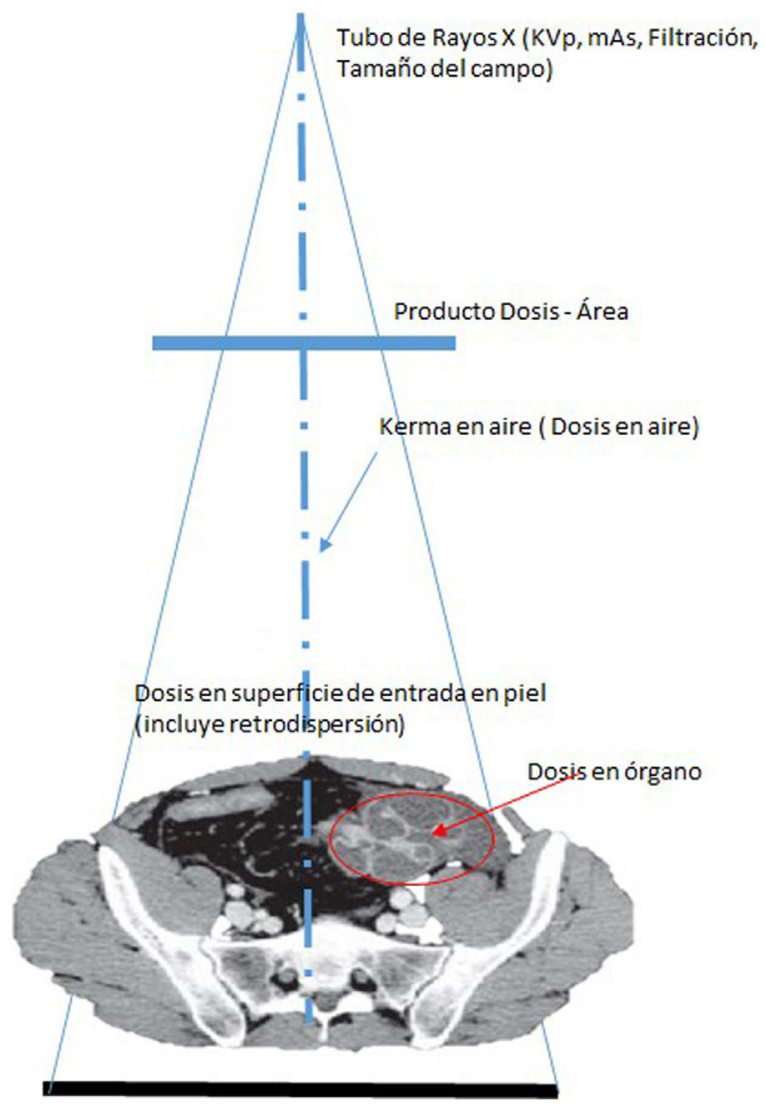

Figura 9 El Kerma y la dosis dependerán de los factores de exposición, $\mathrm{KVp}$, mAs, filtración total (inherente más agregada) y la colimación. La dispersión de los rayos $\mathrm{X}$, debido al efecto Compton, es la responsable de la dosis ocupacional en los médicos intervencionistas.

determinado paciente (por ejemplo, la edad) puede diferir de aquel que pertenece a la población ocupacionalmente expuesta en la que se basan los valores de $\mathrm{w}_{\mathrm{T}}$. La unidad de dosis efectiva es $\mathrm{J} / \mathrm{kg}$, o Sievert (Sv).
La dosis efectiva es una cantidad derivada, o construcción matemática, y no una cantidad física y medible. La cantidad calculada de "dosis efectiva" se usa apropiadamente en protección radiológica para asegurar que no se excedan los límites de exposición para los trabajadores ocupacionalmente expuestos. Los principales usos de la dosis efectiva son la evaluación prospectiva de la dosis para la planificación y la optimización de la protección radiológica y la demostración del cumplimiento de los límites de dosis con fines reglamentarios.

En las aplicaciones médicas que utilizan imágenes producidas con rayos $X$, ya sea para diagnóstico o como guía para procedimientos intervencionistas, dado que no es posible medir directamente la dosis absorbida y, por consiguiente, las cantidades de dosis equivalente y dosis efectiva tampoco se pueden evaluar en la práctica. Entonces para exposiciones ocupacionales sus valores están determinados por la monitorización de radiación utilizando magnitudes operativas. Estas son magnitudes dosimétricas definidas para ser usadas en las mediciones de la exposición externa con fines de protección radiológica (vigilancia radiológica individual o de área). Las magnitudes operativas pueden estar relacionadas con magnitudes físicas básicas cuyas unidades se obtienen directamente a través de patrones primarios en los laboratorios nacionales para estandarización, por ejemplo el Kerma en aire para fotones, la afluencia de partículas para neutrones y la dosis absorbida para radiación beta. La vigilancia radiológica individual se realiza comúnmente con dosímetros colocados sobre el cuerpo, y la magnitud operativa definida para esta aplicación considera esta situación. Para la vigilancia radiológica individual la magnitud operativa es el equivalente de dosis personal, $H p(d)$, que es el equivalente de dosis en tejido a una profundidad $d$ en un cuerpo humano debajo de la posición donde se usa el dosímetro individual. Para la vigilancia de la dosis en piel se recomienda $d=0.07 \mathrm{~mm}$ y para la vigilancia de la dosis efectiva $d=10 \mathrm{~mm}$. En los casos de vigilancia de la dosis en cristalino, como para el caso de procedimientos intervencionistas como la hemodinamia, puede ser apropiada una profundidad $\mathrm{d}=3 \mathrm{~mm}$.

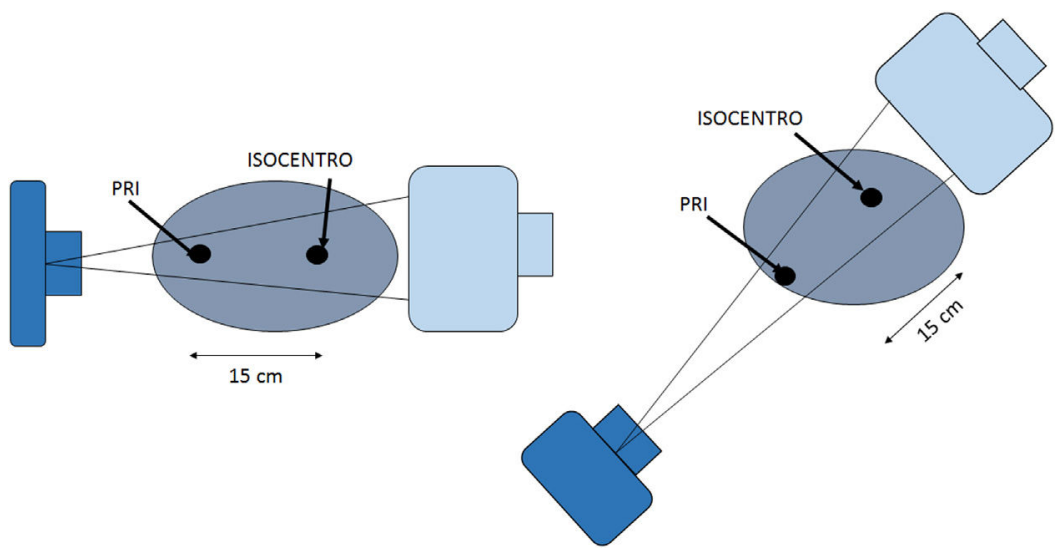

Figura 10 Geometría del punto de referencia intervencionista (PRI) y el isocentro. 


\section{Creative commons}

Creative Commons Reconocimiento-No Comercial-Sin Obra Derivada (CC BY-NC-ND).

\section{Conflicto de intereses}

Ninguno.

\section{Bibliografía}

1. Dance DR, Christofides S, Maidment ADA, McLean ID, Ng KH. (eds). Diagnostic Radiology Physics. Vienna: IAEA; 2014. p. 2-9.

2. Bushberg JT, Sievert JA, Leidholt EM, Boone JM. The essential physics of medical imaging. Philadelphia: Lippincott Williams Wilkins; 2002. p. 97-102.
3. Schueler BA. The AAPM/RSNA physics tutorial for residents: general overview of fluoroscopic imaging. Radiographics. 2000;20:1115-26.

4. Carrol QB. Radiology in the Digital Age. Springfield: Charles C. Thomas Pub; 2011.

5. Papp J. Quality management in the imaging sciences. St LouisMissouri: Elsevier; 2011.

6. ICRP. 1990 Recommendations of the International Commission on Radiological Protection. ICRP Publication 60. Ann. ICRP 21.(1-3).

7. Attix FH. Introduction to radiological physics and radiation dosimetry. Hoboken: John Wiley \& Sons; 1986.

8. ICRP. The 2007 Recommendations of the International Commission on Radiological Protection. ICRP Publication 103. Ann. ICRP 37. (2-4).

9. National Council on Radiation Protection and Measurements. Radiation dose management for fluoroscopically guided interventional medical procedures Report No. 168. Bethesda: NCRP; 2015. 